\title{
Ceramide Dynamics and Prognostic Value in Acute and Subacute Ischemic Stroke: Preliminary Findings in a Clinical Cohort
}

\author{
Marina Buciuc ${ }^{\mathrm{a}}$, Vlad C. Vasile ${ }^{\mathrm{b}, \mathrm{c}}$, Gian Marco Conte ${ }^{\mathrm{d}}$, Eugene L. Scharf ${ }^{\mathrm{a}, \mathrm{e}}$
}

\begin{abstract}
Background: Ceramides are implicated in sphingolipid signaling. Elevated ceramide levels have been associated with increased cardiovascular risk, but information on their role in acute ischemic stroke (AIS) is limited. The purpose of this study is to investigate the temporal dynamics of ceramide levels in AIS and assess their prognostic utility for long-term outcomes.
\end{abstract}

Methods: This is a prospective pilot study of patients with AIS admitted to Mayo Clinic within $12 \mathrm{~h}$ of last known well (LKW). Ceramides were assessed by liquid chromatography mass spectrometry at two time points: T1 (within $12 \mathrm{~h}$ of LKW) and T2 (1 - 7 days from LKW). Wilcoxon signed rank test was used to compare paired ceramide levels and ratios. Ordinal logistic regression was used for assessment of associations with long-term outcomes.

Results: Twenty-three patients met inclusion criteria (median (range)): age (76 years (45 - 95)); body mass index (25.6 (20.5 46.6)); National Institutes of Health Stroke Scale (NIHSS) score (5 $\left(0\right.$ - 27)); infarct volume $\left(1.4 \mathrm{~cm}^{3}(0.0\right.$ - 36.5)). Long-chain ceramides increased between $\mathrm{T} 1$ and $\mathrm{T} 2$ whereas very-long chain ceramides decreased, $\mathrm{P}<0.05$. Upon stratification of patients by prior statin exposure, increase in long-chain ceramide level was present only in statin-naive patients. Greater neurological disability at follow-up was associated with higher ceramide score, $\mathrm{C}(18: 0) / \mathrm{C}(24: 0)$ ratio and higher levels of glycated hemoglobin.

Conclusions: Long-chain and very-long-chain ceramide are actively implicated in pathologic processes in acute and subacute phases of stroke, with their dynamics being inversely related and potentially

Manuscript submitted September 1, 2020, accepted October 31, 2020

Published online December 9, 2020

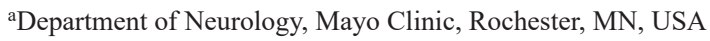

bepartment of Laboratory Medicine and Pathology, Mayo Clinic, Rochester, MN, USA

'Department of Cardiovascular Medicine, Mayo Clinic, Rochester, MN, USA ${ }^{\mathrm{d}}$ Department of Radiology, Mayo Clinic, Rochester, MN, USA

${ }^{\mathrm{e} C}$ Corresponding Author: Eugene L. Scharf, Department of Neurology, Mayo Clinic, College of Medicine and Science, 200 First Street S.W., Rochester, MN 55905, USA. Email: scharf.eugene@mayo.edu

doi: https://doi.org/10.14740/jnr633 modulated by statin therapy. Ceramide levels and ratios might be useful for prognosis of long-term neurological outcomes.

Keywords: Ischemic stroke; Transient ischemic attack; Cerebrovascular disease/stroke; Ceramide; Biomarker; Cell signaling

\section{Introduction}

Sphingolipids (SPLs), biologically active components of cell membranes, are involved in multiple functions such as cell proliferation, differentiation, apoptosis, adhesion, migration and other mechanisms of acute stress response [1]. Ceramides are the chief substrates of the sphingolipid cell signaling pathway, and dynamics in their plasma levels have been associated with a number of metabolic [2] and vascular conditions [3], as well as neurodegenerative disorders [4]. Association between elevated plasma concentrations of ceramides and increased risk of major adverse cardiovascular events, as well as increased risk of mortality following cardiovascular event has been reported by multiple centers $[5,6]$; risk-predicting ceramide scores have also been proposed and are currently used clinically for cardiovascular risk stratification [7, 8]. Elevated levels of specific ceramides and SPLs have been also associated with small vessel ischemic disease and white matter hyperintensity $[9,10]$. Several preclinical and clinical studies reported changes in plasma and/or cerebral levels of ceramides in acute stroke [11-13], with a recent case-control study proposing cut-off levels of specific ceramides as supplemental predictors of risk and severity of ischemic stroke [14]. Ceramides are evidently important players in the pathogenesis of acute cerebral ischemia; however, little is known about SPL and ceramide dynamics between acute and subacute stroke phases, which is crucial for applicability of proposed predictors as well as association between plasma ceramide levels and long-term neurological outcomes.

In this exploratory study our primary aims are: 1) to assess the dynamics of SPL and ceramide levels in acute $(\leq 12$ $\mathrm{h}$ from last known well (LKW)) and subacute phases of acute ischemic stroke (AIS); 2) to evaluate association between SPL and ceramide levels and long-term neurological outcomes. Our secondary aim is to test sensitivity and specificity of proposed predictors of risk and severity of ischemic stroke [14] in 
our cohort of patients, and whether the performance of these predictors is time-dependent.

\section{Materials and Methods}

\section{Study design and participants}

We conducted a prospective longitudinal observational study using patients who presented with AIS or transient ischemic attack (TIA) symptoms to the Mayo Clinic Hospital (Saint Mary's campus) in Rochester, $\mathrm{MN}$ or referring health system emergency room. All patients were screened and enrolled between November 1, 2018 and December 1, 2019. We identified patients who met the following inclusion criteria: 1) presented to the hospital within $12 \mathrm{~h}$ of symptom onset or LKW; 2) had National Institutes of Health Stroke Scale (NIHSS) [15] score $\geq 2$ or NIHSS score $=0$ and ABCD2 score $\geq 4[16,17]$ for suspected TIA on admission; 3 ) initial non-contrast computed tomography (CT) head excluded the presence of primary intracerebral hemorrhage or subarachnoid hemorrhage. Patients who were $<18$ years old, had fever $>38.0^{\circ} \mathrm{C}$ and/or white blood cell count $>16,000$ cells $/ \mu \mathrm{L}$, had a history or suspicion of active endocarditis, active malignancy, inflammatory vasculopathy (e.g., Moya-Moya, Susac's syndrome), systemic inflammatory disease, connective tissue disease, hypercoagulable state, seizure at presentation, or pregnancy were excluded.

Twenty-seven patients met our inclusion criteria. On admission patients underwent emergency neurological evaluation according to accepted practice standards [18, 19]. Dynamics of SPL and ceramide levels were investigated in pooled venous blood acquired by phlebotomy. The first blood draw (T1) was performed within $12 \mathrm{~h}$ of LKW and intended to measure SPL and ceramide levels during AIS. The second time point (T2) for blood sample collection was drawn at least 24 $\mathrm{h}$ but not longer than 7 days after the symptom onset or LKW, and was intended to measure SPL and ceramide levels during subacute phase of cerebral ischemic event. Demographic and clinical characteristics as well as relevant laboratory markers were abstracted from the medical record. Screening logs were kept. Mechanism of acute ischemic event and modified Rankin scale (mRS) score $[20,21]$ for neurological disability 90 days after the event have been determined by retrospective chart review conducted by a blinded to imaging and laboratory data board certified vascular neurologist. Long-term neurological outcomes were measured by $\mathrm{mRS}$ score at 90 days after the event.

\section{Standard protocol approvals, registrations and patient in- formed consents}

This study has been approved by the Mayo Clinic Institutional Review Board, and all participants and/or their proxies signed a written informed consent form before taking part in any research activities in accordance with the Declaration of Helsinki. Due to time-sensitivity of the sample acquisition a deferred consent was approved for the present study by the Mayo
Clinic Institutional Review Board, which allowed collection of a blood sample from a qualifying candidate when a written informed consent was not possible to obtain within $12 \mathrm{~h}$ of LKW. In those cases written informed consent was still required from the participants and/or their proxies after the sample collection before any data abstraction and/or analyses were performed; otherwise, the collected samples were destroyed.

\section{Biochemical analysis}

Venous blood ( $3 \mathrm{~mL}$ ) were collected by phlebotomy into chilled EDTA-containing tubes, centrifuged for plasma separation, aliquoted in 1.5-mL tubes, snap-frozen and immediately stored at $-80{ }^{\circ} \mathrm{C}$ until further analyses [22]. Quantitative assays for levels of sphingosine, sphinganine, sphingosine-1-posphate, N-palmitoyl-sphingosine (C16:0-ceramide), N-stearoyl-sphingosine (C18:0-ceramide), N-arachidoyl-D-erythro-sphingosine (C20:0ceramide), N-docosanoyl-sphingosine (C22:0-ceramide), Nnervonoyl-sphingosine (C24:1-ceramide) and N-lignoceroylsphingosine (C24:0-ceramide) were performed by Mayo Clinic Metabolomics by previously described technique [4, 23]. Briefly a $25 \mu \mathrm{L}$ aliquot of plasma was spiked with internal standards mixture prior to undergoing extraction. Data acquisition was done using select ion monitor (SRM) after chromatographic separation and electron ionization on the Thermo Scientific TSQ Quantiva triple-stage quadrupole mass spectrometer (West Palm Beach, FL) coupled with a Waters Acquity UPLC system (Milford, MA). Concentrations of each analyte were calculated against each perspective calibration curve. Coefficient of variation of a healthy control plasma analyzed with each batch of 40 samples over 1 month period are $6.3 \%, 6.2 \%$, $3.1 \%, 5.0 \%, 5.7 \%, 3.2 \%, 4.9 \%$ and $3.3 \%$ for sphingosine, sphingosine-1-phosphate, C16:0-ceramide, C18:0-ceramide, C20:0-ceramide, C22:0-ceramide, C24:1-ceramide and C24:0ceramide, respectively. Upon acquisition of quantitative data ceramide scores at T1 and T2 were calculated for each patient as previously described $[5,7]$. Briefly, a combined pool of angiography patients $(n=477)$ and healthy donors $(n=168)$ was used to establish median value for each ceramide, ceramide ratio and the quartiles. If the value of C16:0-ceramide, C18:0ceramide, or C24:0-ceramide was above median 1 point was added, if the values of above mentioned ceramides were in the fourth quartile, additional point was added to each value. Similarly, points were added for each ratio C16:0/C24:0, C18:0/ $\mathrm{C} 24: 0$, and C24:1/C24:0 being above median or in the fourth quartile.

\section{Neuroimaging analysis}

For each patient, manual segmentations of the infarct areas were obtained using the software RIL-Contour [24]. Areas of restricted diffusion were segmented on diffusion-weighted imaging (DWI) images without using a priori thresholds. To confirm true restricted diffusion and avoid the T2 shine through phenomena [25], apparent diffusion coefficient (ADC) maps were used as a reference to look for a corresponding area of 
low signal. The final segmentation included only areas that presented as hyperintense on DWI and hypointense on ADC maps. In cases where magnetic resonance imaging (MRI) was not performed $(n=6)$, infarct areas were calculated using noncontrast-enhanced CT scans.

\section{Statistical analysis}

JMP Pro 14 statistical software package was used for data analysis (https:/www.jmp.com/en_us/software/predictive-analytics-software.html). Due to the actual sample size $<25$ and not normally distributed data nonparametric methods were implemented for statistical analyses. The Wilcoxon rank sum test was used to compare continuous variables; while Chi-square or Fisher's exact tests were used for categorical variables such as gender. The primary endpoint was difference in median (95\% confidence interval) levels of ceramides and ceramide score between T1 to T2; the estimates and 95\% confidence intervals were calculated using smoothed empirical likelihood methods for quantiles [26]. Statistical significance of difference in levels of paired measurements was assessed by twosided Wilcoxon signed rank test with predetermined level of significance defined as any $\alpha$ error of $<0.05$. Association between long-term neurological outcome ( $\mathrm{mRS}$ score at 90 days follow-up) was first assessed by conducting univariate ordinal logistic regression analysis using each demographic, clinical and laboratory characteristics as independent variables. Variables that were not normally distributed were log transformed prior to analysis. Independent variables that were found statistically significant after univariate analysis were included in multivariate ordinal logistic regression model. Area under the receiver operating characteristic curve (AUROC) was calculated for sensitivity/specificity analyses. Figures were generated in R software version 3.4.2 [27]. No formal power calculation was completed for enrollment, it was estimated that approximately 25 patients would provide adequate measures of central tendency for powering larger studies.

\section{Results}

\section{Demographic, clinical and laboratory characteristics}

A total of 23 patients were included for the final analyses: two patients were excluded due to failure to obtain one of the two required blood samples; two patients were excluded due to absence of signs of justifiable cerebral ischemia during the diagnostic workup (e.g., absence of areas of restricted diffusion on DWI MRI scan, substantial stenosis of carotid arteries). Demographic, clinical and laboratory characteristics of the study participants are summarized in Table 1.

We stratified the patients by prior to the event therapy with statins due to its possible confounding effects on ceramide and SPL level in the light of their interaction with lipid metabolism [28]. Patients that were statin-naive prior to admission had significantly higher levels of low-density lipoproteins, but did not differ from statin-exposed patients otherwise. Twenty out of 23
$(87 \%)$ patients received statin therapy while inpatient.

\section{Temporal difference in SPL and ceramide levels}

Levels of sphingosine, sphingosine-1-posphate, and sphinganine did not differ between $\mathrm{T} 1$ and $\mathrm{T} 2$ in all as well as stratified by prior statin exposure patients, $\mathrm{P}>0.05$. Total ceramide levels reduced between $\mathrm{T} 1$ and $\mathrm{T} 2, \mathrm{P}=0.0012$; upon stratification by statin-exposure status this change remained significant only for statin-exposed patients, $\mathrm{P}=0.0098$. Temporal differences in ceramide levels, ceramide ratios and ceramide score are summarized in Figures 1, 2 and Table 2. Long-chain C18:0ceramide significantly increased between acute and subacute phases of AIS, whereas very-long-chain ceramides C22:0 and C24:0 decreased (Fig. 1). Upon stratification by prior statin exposure, long-chain C18:0-ceramide remained significantly increased only in statin-naive group whereas very-long-chain ceramide levels C20:0, C22:0 and C24:0 significantly decreased in statin-exposed group and to a lesser extent C22:0 decreased in statin-naive participants (Fig. 2 a, c). Ceramide ratios also differed between the time points, but to a greater extent in statin-naive participants (Fig. 2b, d). Ceramide scores did not differ between the two time points in all as well as stratified by prior statin exposure patients.

\section{Association of ceramide levels and long-term neurological outcomes}

Univariate logistic regression showed association of $\mathrm{mRS}$ with the following variables: C18:0 levels at T1 and T2, C18:0/ $\mathrm{C} 24: 0$ ratio at $\mathrm{T} 1$ and $\mathrm{T} 2, \mathrm{C} 16: 0 / \mathrm{C} 24: 0$ ratio at $\mathrm{T} 2$, ceramide score at T2 and glycated hemoglobin (HbA1c) levels. Since ceramide score is directly associated with ceramide levels and ratios it was used in a separate multivariate ordinal logistic model with HbA1c. Greater ceramide score and HbA1c levels were both associated with greater neurological disability, $\mathrm{P}=$ 0.005 and $\mathrm{P}=0.019$, respectively.

Since $\mathrm{C} 18: 0$ is incorporated in $\mathrm{C} 18: 0 / \mathrm{C} 24: 0$ ratio, the ratio was used instead in the multivariate model. Separate multivariate ordinal regression models were performed for T1 and T2.

Multivariate regression model including C18:0/C24:0 ratio at $\mathrm{T} 1$ and $\mathrm{HbA} 1 \mathrm{c}$ showed that both variables were associated with $\mathrm{mRS}$ score, $\mathrm{P}=0.0108$ and $\mathrm{P}=0.0229$, respectively. Multivariate regression model including $\mathrm{C} 18: 0 / \mathrm{C} 24: 0$ and $\mathrm{C} 16: 0 / \mathrm{C} 24: 0$ ratios at $\mathrm{T} 2$ and $\mathrm{HbA} 1 \mathrm{c}$ showed that only $\mathrm{C} 18: 0 /$ $\mathrm{C} 24: 0$ ratio was associated with $\mathrm{mRS}$ score, $\mathrm{P}=0.0119$.

\section{Validation of proposed cut-off values for prediction of risk and severity of stroke}

We applied the following cut-off values proposed by Gui et al [14] for auxiliary diagnosis of stroke at two time points: C16:0-ceramide $(0.26 \mu \mathrm{mol}), \mathrm{C} 22: 0$-ceramide $(0.62 \mu \mathrm{mol})$, and $\mathrm{C} 24: 0$-ceramide $(2.27 \mu \mathrm{mol})$.

At T1 $(<12 \mathrm{~h}$ from LKW) in cohort of 25 patients (AIS (n 
Table 1. Demographic, Clinical and Laboratory Characteristics of the Study Participants

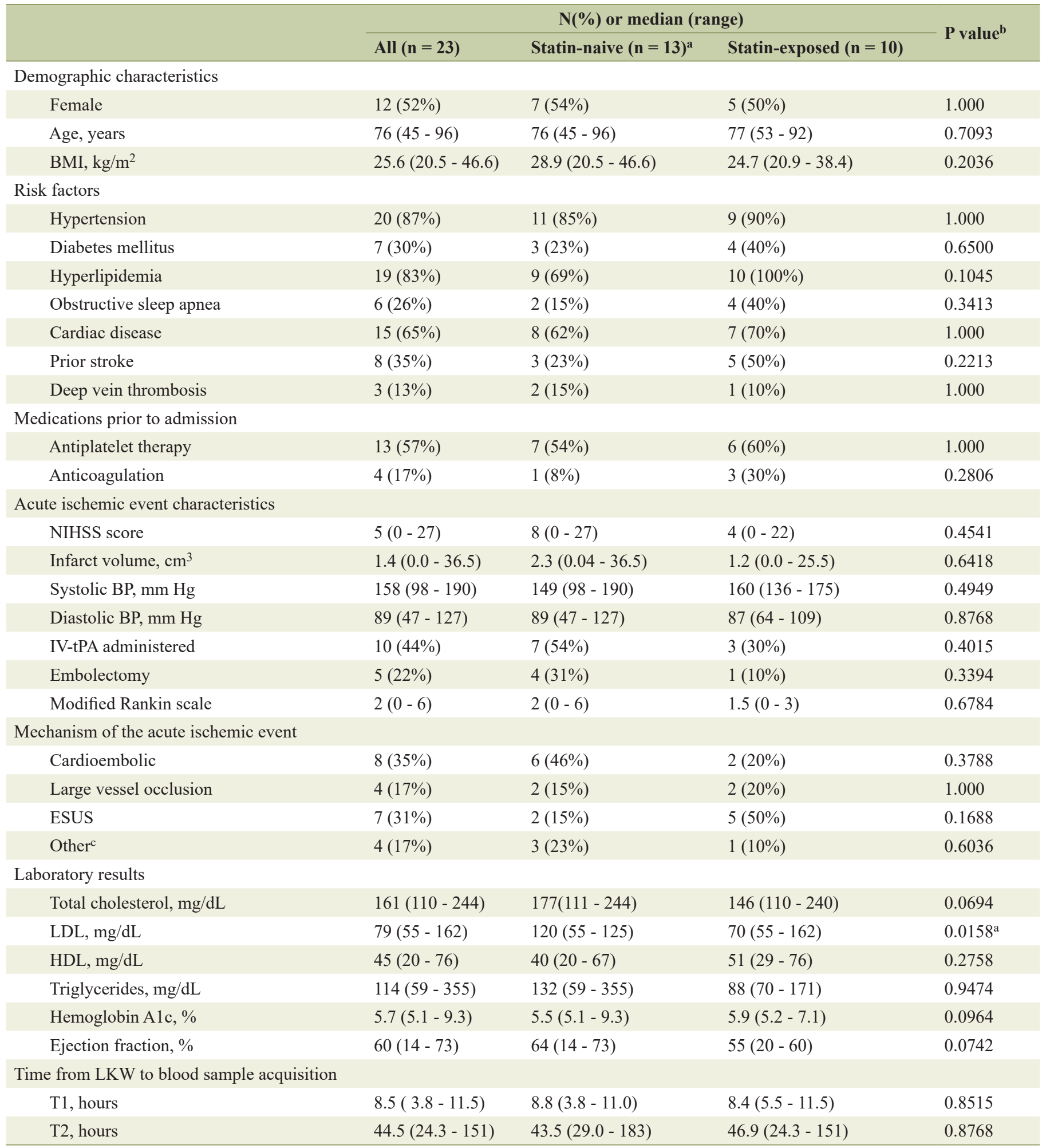

LKW: last known well; BMI; body mass index; NIHSS: National Institutes of Health Stroke Scale; BP: blood pressure; IV-tPA: intravenous tissue plasminogen activator; ESUS: embolic stroke of undetermined source; LDL: low-density lipoprotein; HDL: high-density lipoprotein; T1: time point 1; T2: time point 2. aStatin-naive: participants were not on statin therapy prior to admission. ${ }^{b}$ For categorical variables, $P$ values are from Fisher's exact test; for continuous, from Wilcoxon rank sum test. 'Other mechanisms: dissection $(n=1)$, hypotension $(n=1)$, transient ischemic attack $(n=1)$, small vessel occlusion $(n=1)$. 


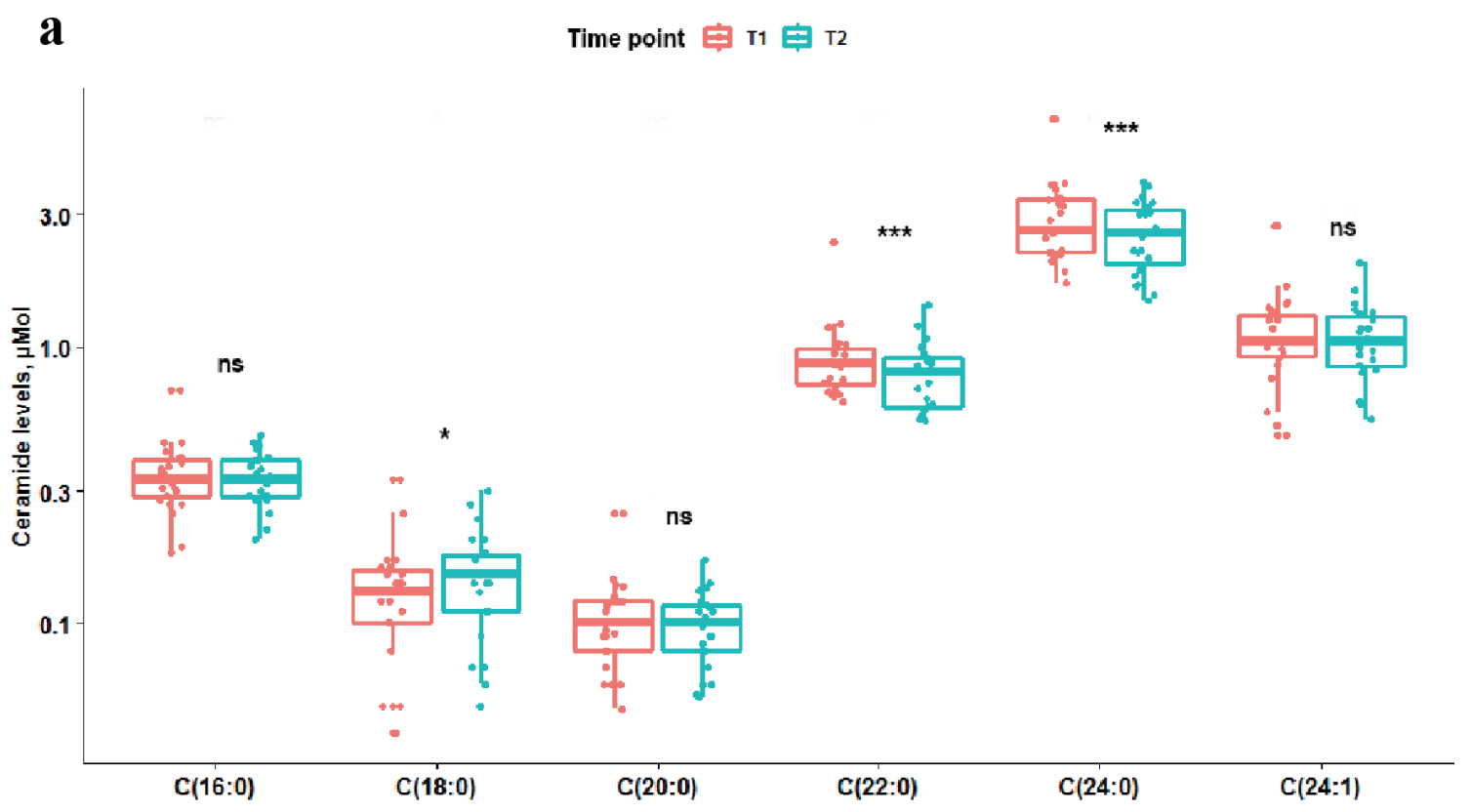

b

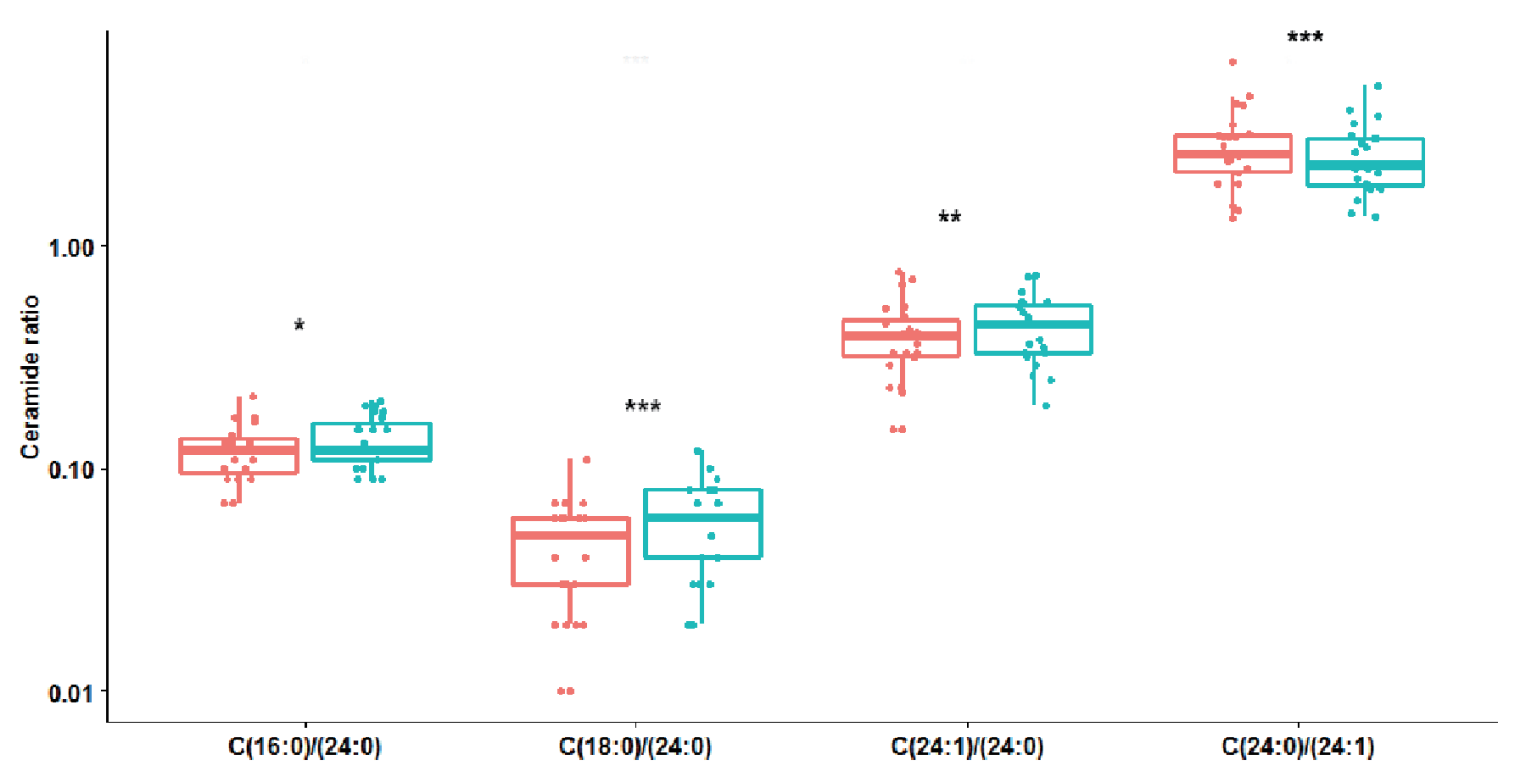

Figure 1. Box plots showing difference in ceramide levels and ratios between two time points. Coral box plots represent circulating plasma ceramide levels (a) or ratios (b) at time point $1(<12 \mathrm{~h}$ from the symptom onset), and teal box plots represent ceramide levels or ratios at time point 2 (> $24 \mathrm{~h}$ from the symptom onset). The line in box plots represents the median, interquartile range and the whiskers correspond to minimum and maximum values. Wilcoxon signed rank test results are displayed above each comparison. ns: not significant, $\mathrm{P} \geq 0.05 .{ }^{*} 0.01 \leq \mathrm{P}<0.05 .{ }^{* *} 0.001 \leq \mathrm{P}<0.01 .{ }^{* * *} \mathrm{P}<0.001$.

$=22)$, TIA $(\mathrm{n}=1)$, functional spell $(\mathrm{n}=2))$ proposed cut-off values were $\mathrm{C} 16: 0$ and $\mathrm{C} 22: 0$ cut-off values were quite sensitive, but not specific. At T2 ( $>24 \mathrm{~h}$ of LKW) the proposed cutoff values were able to diagnose stroke with slightly improved specificity, but at cost of sensitivity (Table 3A).

We applied the following cut-off values proposed by Gui et al [14] for prediction of moderate-severe stroke defined as
NIHSS $\geq 6$ at two time points: $\mathrm{C} 16: 0$-ceramide $(0.30 \mu \mathrm{mol})$, C22:0-ceramide $(0.71 \mu \mathrm{mol})$, and C24:0-ceramide $(2.38$ $\mu \mathrm{mol})$.

At T1 in cohort of 22 patients with confirmed stroke, 9/22 (41\%) having NIHSS $\geq 6, \mathrm{C} 16: 0$ and $\mathrm{C} 22: 0$ proposed cut-off values were moderately sensitive, but not specific with even worse performance in subacute phase of stroke (Table 3B). 

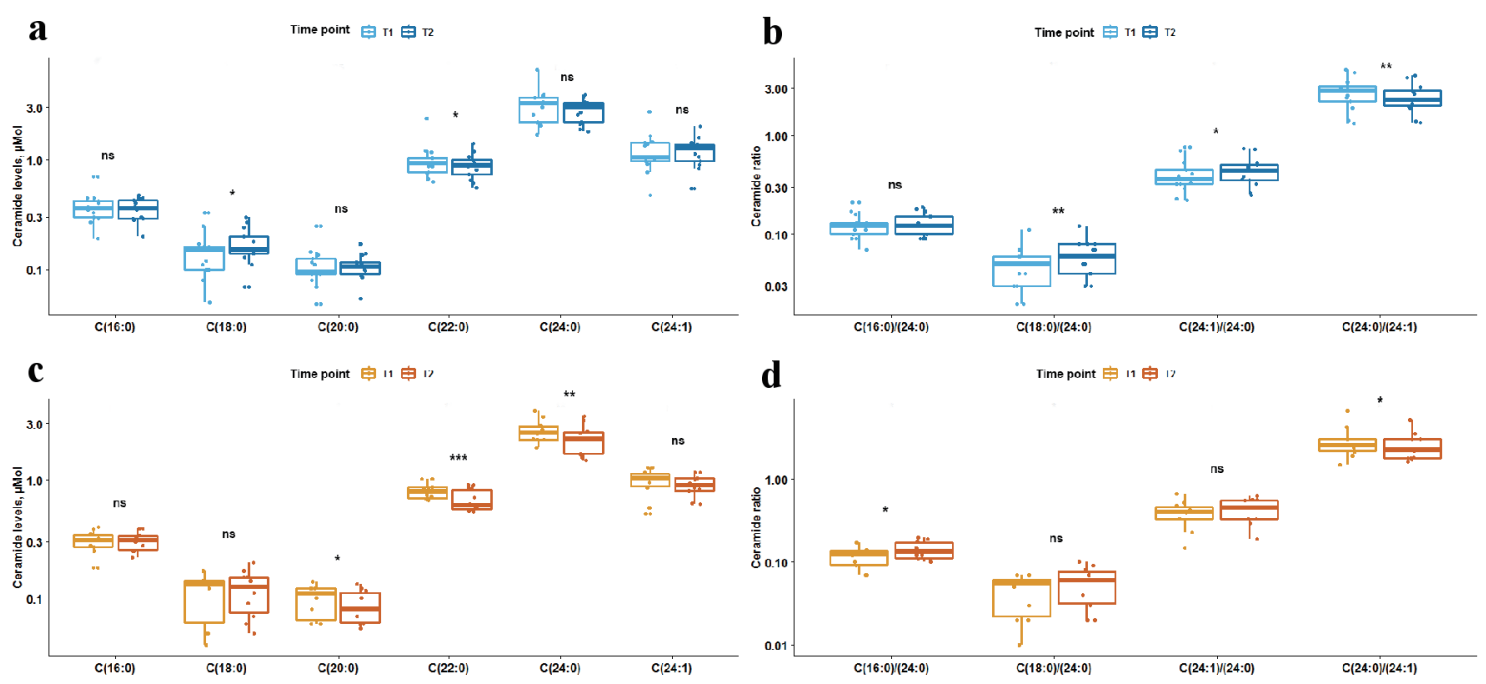

Figure 2. Box plots showing difference in levels of ceramides and ceramide ratios between two time points stratified by prior statin exposure. Light blue and orange box plots represent circulating plasma ceramide levels $(a, c)$ or ratios (b, d) at time point 1 , and dark blue and orange box plots represent ceramide levels or ratios at time point 2 . Panels (a) and (b) show data for statinnaive participants; panels (c) and (d) show data for participants with prior statin exposure. The line in box plots represents the median, interquartile range and the whiskers correspond to minimum and maximum values. Wilcoxon signed rank test results are displayed above each comparison. ns: not significant, $\mathrm{P} \geq 0.05$. ${ }^{*} 0.01 \leq \mathrm{P}<0.05$. ${ }^{* *} 0.001 \leq \mathrm{P}<0.01$. ${ }^{* *} \mathrm{P}<0.001$.

\section{Discussion}

Unraveling pathogenic mechanisms underlying acute cerebral ischemia as well as their dynamics over time are essential for better prevention, management and recovery of stroke and TIA patients. In this study we report that long-chain and very-longchain ceramides have inverse dynamics in acute vs. subacute phases of cerebral ischemia with levels of long-chain ceramides increasing over time and very-long-chain ceramides decreasing. Prior exposure to statins might have a role in alteration of these dynamics by attenuation of the rise in long-chain ceramide levels. We also report that higher ceramide score, $\mathrm{C} 18: 0 / \mathrm{C} 24: 0$ ratio and $\mathrm{HbA} 1 \mathrm{c}$ levels are negative prognostic factors of neurological disability at 90 days after the event.

Our finding of increased circulating plasma levels of longchain ceramides, in our case specifically C18:0-ceramide, is in keeping with previously published preclinical and clinical studies $[12,14]$. Murine studies showed that plasma levels of long-chain ceramides even though rising in the first hours of AIS reached their peaks at least $24 \mathrm{~h}$ after ischemic insult [12] with similar findings in the ischemic tissue [11, 29, 30]. Our study is the first report to date which now recapitulates these animal findings in humans. Noteworthy, the rise in ceramide levels was previously observed only in animal models with successful reperfusion versus complete or more prolonged occlusion of the cerebral blood flow [29-31]; whether same relationship exists in clinical patients is yet to be determined. Long-chain ceramides are involved in apoptotic pathways, inflammation and JNK signaling activated by cerebral ischemia and leading to mitochondrial dysfunction [29, 32]. Therefore, rise in ceramide levels is most likely the sign of ongoing inflammation and tissue damage which is still happening after reperfusion and in subacute phases of stroke. Interestingly, we found temporal rise in C18:0 levels extending to subacute phase of stroke only in statin-naive patients. Daily atorvastatin has been shown to reduce the risk of a recurrent stroke in patients who experienced AIS through predominantly reduction in low-density lipoprotein (LDL) cholesterol levels [28]. Ceramide levels have also been shown to decrease with statin use $[33,34]$. Murine study showed that administration of atorvastatin within $1 \mathrm{~h}$ of AIS prevented the rise of long-chain ceramides levels both at 3 and $24 \mathrm{~h}$ post-occlusion. Hence, statins might serve an important role in reduction of inflammation and tissue damage during AIS.

Contrary to the rising long-chain ceramide levels, we observed decreasing over time levels of very-long-chain ceramides which is also consistent with reported results $[12,13]$. Production of long-chain and very-long-chain ceramides is regulated by different ceramide synthases [35]; in contrast to long-chain ceramides, very-long-chain ceramides have been linked to cell proliferation and potentially neuroprotective effects during anoxia $[35,36]$. Reduction in very-long-chain ceramide levels was more prominent in statin-exposed patient though still statistically significant for $\mathrm{C}(22: 0)$-ceramide in statin-naive patients which is discordant with the murine study where a decrease in very-long-chain ceramide levels was not observed upon administration of atorvastatin [12]. One of the possible explanations of the difference in clinical and murine findings can be linked to the time of statin administration. Whereas in the murine study atorvastatin was administered $1 \mathrm{~h}$ post-ischemia, patients in our study have been on statintherapy prior to admission and inpatient administration of atorvastatin exceeded $1 \mathrm{~h}$ post-occlusion in all cases. Additionally, due to limited studies in humans it is unclear whether same ceramide synthase types are equally expressed and enriched in the same tissues types in humans as they are described in 
Table 2. Median Difference in Levels of Circulating Ceramides Between the Two Time Points

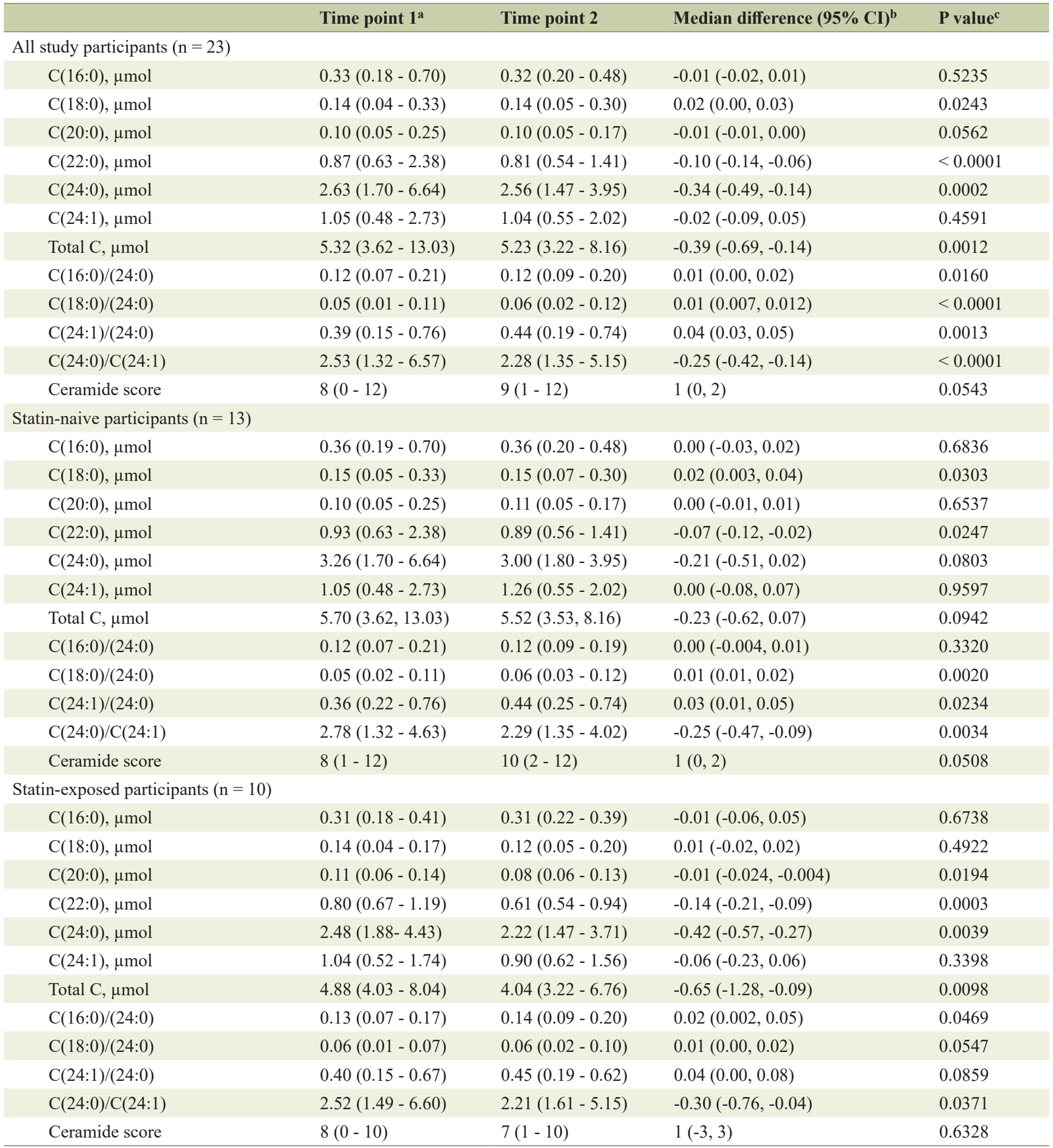

$\mathrm{Cl}$ : confidence interval; C: ceramide. ${ }^{a}$ Data shown for time point 1 and 2 are median (range). ${ }^{\text {b }}$ Median difference and $95 \%$ confidence intervals are from smoothed empirical likelihood quantiles. ${ }^{\mathrm{P}}$ values shown are from Wilcoxon signed rank test, $\mathrm{P}<0.05$ was considered statistically significant.

the murine models. There is some evidence in discordance of ceramide synthase enrichment in a specific tissue in mice and clinical phenotype in humans when same ceramide synthase or the levels of ceramide it produces are altered [37]. It is also 


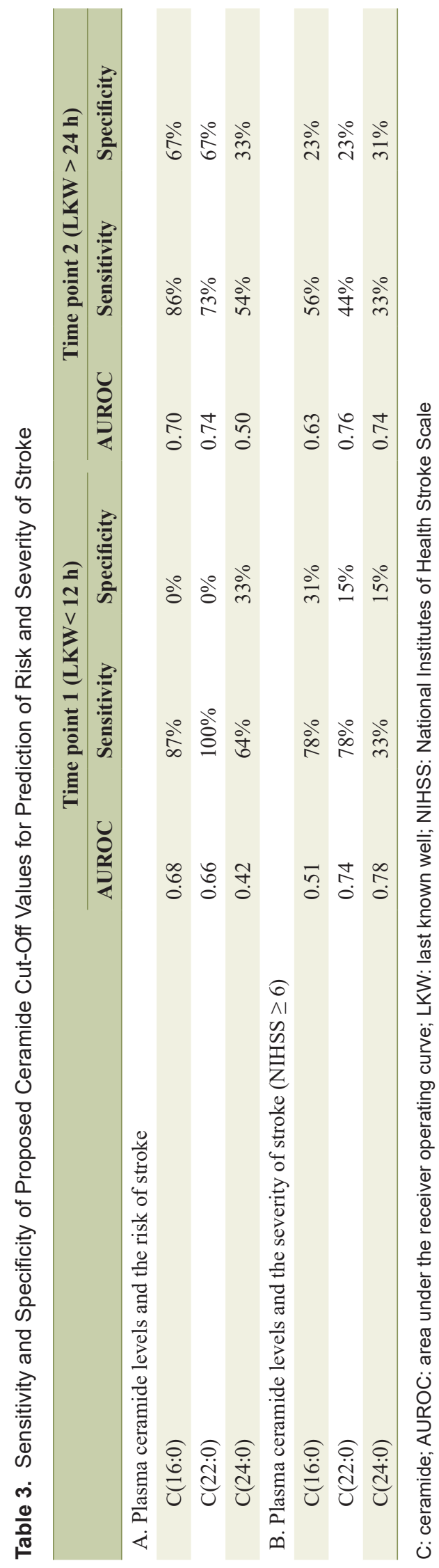

possible that other factors interfered with statin effect on SPL metabolism in statin-naive and statin-exposed patients.

We found an association between higher levels of $\mathrm{HbAlc}$, ceramide score and specific ceramide levels and ratios with worse neurological outcomes. Our findings are consistent with previous reports on hemoglobin A1c levels [38-40] as well as association of elevated C18:0-ceramide levels with poor outcomes for stroke patients [41]. Rather than individual ceramide level dynamics we found the $\mathrm{C} 18: 0 / \mathrm{C} 24: 0$ ratio to be the stronger prognostic factor as it reflects the dynamics of both classes of ceramides that have undergone temporal change, long-chain and very-long chain. It is important to mention that $\mathrm{C} 18: 0 / \mathrm{C} 24: 0$ ratio was strongly associated with poor neurological outcomes during both, acute and subacute phases of ACI suggesting that C18:0/C24:0 may be a good lipidomic biomarker. Higher ceramide score was also associated with greater neurological disability at 90 days post-ACI which might expand its utility beyond cardiovascular risk assessment $[5,8]$. Whereas not found significant in our cohort other clinical characteristics such as the subtype of ischemic stroke, particularly cardioembolic strokes, have been associated with increased mortality [42] and therefore further studies on prognostic biomarkers are warranted.

It is unclear what caused the elevation of ceramide levels in acute and subacute phases of AIS: de novo synthesis or demyelination of sphingomyelin? We did not observe changes in sphinganine, sphingosine and sphingosine-1-phosphate levels to support de novo synthesis of ceramides or their production through salvage pathways $[12,43]$; therefore, it is possible that the majority of ceramides were the product of demyelination; however, we did not formally assess it.

Another possibility is that acute stress due to ischemia lead to a shift in ceramide synthase activity favoring production of long-chain ceramides versus very long-chain. Preferentially increased activity of ceramide synthase 1 (CerS1) would explain the rise of C18:0-ceramide levels only and no other long-chain ceramides as it is specific for C18:0-ceramide production [44].

As a secondary aim of our study we assessed the validity and utility of proposed cut-off values for ceramides for prediction of stroke and severity [5] in our cohort. In our experience in acute phase of stroke some proposed cut-off levels were quite sensitive, but not specific for stroke; specificity improved in subacute phase of stroke. Long-chain ceramide C16:0 cutoff value was the most useful whereas very-long chain ceramide cut-off levels had little clinical utility potentially because we saw an association represented by AUROC with the decrease in these ceramide levels in our cohort rather than an increase. Ceramide $\mathrm{C} 16: 0$ levels might be useful in cases when diagnosis is not clear and imaging is not available; however, in acute setting its utility is limited.

The proposed cut-off values performed poorly in severity of stroke prediction with even worse sensitivity and specificity in the subacute phase. Ethnic differences in metabolic profiles might be responsible for differences in performance since the referring cohort is Chinese and all patients in our study are Caucasian. Another explanation of differences in performance could be related to the fact that the reference cohort included only patients with the first ever stroke whereas a third of pa- 
tients from our cohort had a history of AIS or TIA.

Prospective design, adherence to strict inclusion/exclusion criteria, paired plasma samples as well as ample clinical and laboratory phenotyping of the participants are the strengths of this study. Our findings, however, need to be interpreted with caution due to a number of limitations. A modest sample size reduced the power of the study and our ability to detect possibly significant differences. Since all the participants were Caucasian it reduces generalizability of our results to other ethnicities. Lastly, we did not have a matched healthy control group to assess the magnitude of fluctuation of ceramide levels in AIS patients compared to healthy controls.

In the current era of clinical trials assessing acute stroke management it is tantamount to explore the pathogenesis of AIS to direct the development of novel therapies and prevention tools. Ceramides are highly involved in the pathological processes occurring during, after, and, most likely, before AIS; therefore, understanding of the ways how to manage their levels might lead to prevention of poor outcomes and/or provide adjuvant therapies [45].

\section{Acknowledgments}

The authors extend their appreciation to the patients and their families who gave their time and effort to participate in the study, to Amy M. Headlee, our certified clinical research coordinator, and to Bradley J. Erickson, MD, PhD, for his assistance with neuroimaging analysis.

\section{Financial Disclosure}

This study has been funded by the grants from Mayo Clinic Center for Clinical and Translational Science (UL1TR002377) and the American Brain Foundation. These grants served for the design and conduct of the study, collection, management and analysis of the collected data. The sponsor had no role in study design; collection, analysis and interpretation of data; writing the report; or in the decision to submit the article for publication.

\section{Conflict of Interest}

Dr. Buciuc, Dr. Vasile, Dr. Conte and Dr. Scharf report no disclosures and conflicts of interest.

\section{Informed Consent}

The informed consents were obtained.

\section{Author Contributions}

Dr. Buciuc and Dr. Scharf participated in conception and design of the study, patient recruitment, data collection and for- mal analysis. Dr. Vasile assisted with metabolic analysis and Dr. Conte performed neuroimaging analysis of the data. Dr. Buciuc drafted the manuscript and figures; all the authors reviewed the manuscript for intellectual content, edited sections matching their area of expertise, have seen and approved the final version. Dr. Scharf provided funding for the study and supervised the study.

\section{Data Availability}

Anonymized data are available from the corresponding author upon request from any qualified investigator for purposes of replicating procedures and results.

\section{References}

1. Mao C, Obeid LM. Ceramidases: regulators of cellular responses mediated by ceramide, sphingosine, and sphingosine-1-phosphate. Biochim Biophys Acta. 2008;1781(9):424-434.

2. Haus JM, Kashyap SR, Kasumov T, Zhang R, Kelly KR, Defronzo RA, Kirwan JP. Plasma ceramides are elevated in obese subjects with type 2 diabetes and correlate with the severity of insulin resistance. Diabetes. 2009;58(2):337-343.

3. Borodzicz S, Czarzasta K, Kuch M, Cudnoch-Jedrzejewska A. Sphingolipids in cardiovascular diseases and metabolic disorders. Lipids Health Dis. 2015;14:55.

4. Savica R, Murray ME, Persson XM, Kantarci K, Parisi JE, Dickson DW, Petersen RC, et al. Plasma sphingolipid changes with autopsy-confirmed Lewy Body or Alzheimer's pathology. Alzheimers Dement (Amst). 2016;3:4350.

5. Meeusen JW, Donato LJ, Bryant SC, Baudhuin LM, Berger PB, Jaffe AS. Plasma ceramides. Arterioscler Thromb Vasc Biol. 2018;38(8):1933-1939.

6. Havulinna AS, Sysi-Aho M, Hilvo M, Kauhanen D, Hurme R, Ekroos K, Salomaa V, et al. Circulating ceramides predict cardiovascular outcomes in the populationbased FINRISK 2002 cohort. Arterioscler Thromb Vasc Biol. 2016;36(12):2424-2430.

7. Laaksonen R, Ekroos K, Sysi-Aho M, Hilvo M, Vihervaara T, Kauhanen D, Suoniemi M, et al. Plasma ceramides predict cardiovascular death in patients with stable coronary artery disease and acute coronary syndromes beyond LDL-cholesterol. Eur Heart J. 2016;37(25):19671976.

8. Hilvo M, Meikle PJ, Pedersen ER, Tell GS, Dhar I, Brenner H, Schottker B, et al. Development and validation of a ceramide- and phospholipid-based cardiovascular risk estimation score for coronary artery disease patients. Eur Heart J. 2020;41(3):371-380.

9. Azizkhanian I, Sheth SA, Iavarone AT, Lee S, Kakarla V, Hinman JD. Plasma lipid profiling identifies biomarkers of cerebral microvascular disease. Front Neurol. 2019;10:950.

10. Mielke MM, Syrjanen JA, Bui HH, Petersen RC, Knop- 
man DS, Jack CR, Jr., Graff-Radford J, et al. Elevated plasma ceramides are associated with higher white matter hyperintensity volume-brief report. Arterioscler Thromb Vasc Biol. 2019;39(11):2431-2436.

11. Teppo J, Vaikkinen A, Stratoulias V, Matlik K, Anttila JE, Smolander OP, Poho P, et al. Molecular profile of the rat peri-infarct region four days after stroke: Study with MANF. Exp Neurol. 2020;329:113288.

12. Chao HC, Lee TH, Chiang CS, Yang SY, Kuo CH, Tang SC. Sphingolipidomics Investigation of the Temporal Dynamics after Ischemic Brain Injury. J Proteome Res. 2019;18(9):3470-3478.

13. Fiedorowicz A, Kozak-Sykala A, Bobak L, Kalas W, Strzadala L. Ceramides and sphingosine-1-phosphate as potential markers in diagnosis of ischaemic stroke. Neurol Neurochir Pol. 2019;53(6):484-491.

14. Gui YK, Li Q, Liu L, Zeng P, Ren RF, Guo ZF, Wang GH, et al. Plasma levels of ceramides relate to ischemic stroke risk and clinical severity. Brain Res Bull. 2020;158:122127.

15. Lyden P, Brott T, Tilley B, Welch KM, Mascha EJ, Levine S, Haley EC, et al. Improved reliability of the NIH Stroke Scale using video training. NINDS TPA Stroke Study Group. Stroke. 1994;25(11):2220-2226.

16. Johnston SC, Rothwell PM, Nguyen-Huynh MN, Giles MF, Elkins JS, Bernstein AL, Sidney S. Validation and refinement of scores to predict very early stroke risk after transient ischaemic attack. Lancet. 2007;369(9558):283292.

17. Rothwell PM, Giles MF, Flossmann E, Lovelock CE, Redgrave JN, Warlow CP, Mehta Z. A simple score (ABCD) to identify individuals at high early risk of stroke after transient ischaemic attack. Lancet. 2005;366(9479):2936.

18. Powers WJ, Rabinstein AA, Ackerson T, Adeoye OM, Bambakidis NC, Becker K, Biller J, et al. 2018 Guidelines for the Early Management of Patients With Acute Ischemic Stroke: A Guideline for Healthcare Professionals From the American Heart Association/American Stroke Association. Stroke. 2018;49(3):e46-e110.

19. Powers WJ, Rabinstein AA, Ackerson T, Adeoye OM, Bambakidis NC, Becker K, Biller J, et al. Guidelines for the Early Management of Patients With Acute Ischemic Stroke: 2019 Update to the 2018 Guidelines for the Early Management of Acute Ischemic Stroke: A Guideline for Healthcare Professionals From the American Heart Association/American Stroke Association. Stroke. 2019;50(12):e344-e418.

20. Farrell B, Godwin J, Richards S, Warlow C. The United Kingdom transient ischaemic attack (UK-TIA) aspirin trial: final results. J Neurol Neurosurg Psychiatry. 1991;54(12):1044-1054.

21. van Swieten JC, Koudstaal PJ, Visser MC, Schouten HJ, van Gijn J. Interobserver agreement for the assessment of handicap in stroke patients. Stroke. 1988;19(5):604-607.

22. Wood JT, Williams JS, Pandarinathan L, Courville A, Keplinger MR, Janero DR, Vouros P, et al. Comprehensive profiling of the human circulating endocannabinoid metabolome: clinical sampling and sample storage pa- rameters. Clin Chem Lab Med. 2008;46(9):1289-1295.

23. Blachnio-Zabielska AU, Persson XM, Koutsari C, Zabielski P, Jensen MD. A liquid chromatography/tandem mass spectrometry method for measuring the in vivo incorporation of plasma free fatty acids into intramyocellular ceramides in humans. Rapid Commun Mass Spectrom. 2012;26(9):1134-1140.

24. Philbrick KA, Weston AD, Akkus Z, Kline TL, Korfiatis P, Sakinis T, Kostandy P, et al. RIL-contour: a medical imaging dataset annotation tool for and with deep learning. J Digit Imaging. 2019;32(4):571-581.

25. Burdette JH, Elster AD, Ricci PE. Acute cerebral infarction: quantification of spin-density and T2 shine-through phenomena on diffusion-weighted MR images. Radiology. 1999;212(2):333-339.

26. Chen SX, Hall P. Smoothed empirical likelihood confidence intervals for quantiles. The Annals of Statistics. 1993;1166-1181.

27. Team RC. A language and environment for statistical computing. R Foundation for Statistical Computing, Vienna, Austria. 2012. URL http://www.R-project.org.

28. Amarenco P, Bogousslavsky J, Callahan A, 3rd, Goldstein LB, Hennerici M, Rudolph AE, Sillesen H, et al. High-dose atorvastatin after stroke or transient ischemic attack. N Engl J Med. 2006;355(6):549-559.

29. Yu J, Novgorodov SA, Chudakova D, Zhu H, Bielawska A, Bielawski J, Obeid LM, et al. JNK3 signaling pathway activates ceramide synthase leading to mitochondrial dysfunction. J Biol Chem. 2007;282(35):25940-25949.

30. Yu ZF, Nikolova-Karakashian M, Zhou D, Cheng G, Schuchman EH, Mattson MP. Pivotal role for acidic sphingomyelinase in cerebral ischemia-induced ceramide and cytokine production, and neuronal apoptosis. J Mol Neurosci. 2000;15(2):85-97.

31. Nakane M, Kubota M, Nakagomi T, Tamura A, Hisaki H, Shimasaki H, Ueta N. Lethal forebrain ischemia stimulates sphingomyelin hydrolysis and ceramide generation in the gerbil hippocampus. Neurosci Lett. 2000;296(23):89-92.

32. Tian HP, Qiu TZ, Zhao J, Li LX, Guo J. Sphingomyelinase-induced ceramide production stimulate calcium-independent JNK and PP2A activation following cerebral ischemia. Brain Inj. 2009;23(13-14):1073-1080.

33. Tarasov K, Ekroos K, Suoniemi M, Kauhanen D, Sylvanne T, Hurme R, Gouni-Berthold I, et al. Molecular lipids identify cardiovascular risk and are efficiently lowered by simvastatin and PCSK9 deficiency. J Clin Endocrinol Metab. 2014;99(1):E45-52.

34. Ng TW, Ooi EM, Watts GF, Chan DC, Meikle PJ, Barrett $\mathrm{PH}$. Association of plasma ceramides and sphingomyelin with VLDL apoB-100 fractional catabolic rate before and after rosuvastatin treatment. J Clin Endocrinol Metab. 2015;100(6):2497-2501.

35. Grosch S, Schiffmann S, Geisslinger G. Chain lengthspecific properties of ceramides. Prog Lipid Res. 2012;51(1):50-62.

36. Menuz V, Howell KS, Gentina S, Epstein S, Riezman I, Fornallaz-Mulhauser M, Hengartner MO, et al. Protection of C. elegans from anoxia by HYL-2 ceramide syn- 
thase. Science. 2009;324(5925):381-384.

37. Park JW, Park WJ, Futerman AH. Ceramide synthases as potential targets for therapeutic intervention in human diseases. Biochim Biophys Acta. 2014;1841(5):671-681.

38. Wang H, Cheng Y, Chen S, Li X, Zhu Z, Zhang W. Impact of elevated hemoglobin A1c levels on functional outcome in patients with acute ischemic stroke. J Stroke Cerebrovasc Dis. 2019;28(2):470-476.

39. Zhang G, He M, Xu Y, Li X, Cai Z, Guo Z, Meng P, et al. Hemoglobin A1c predicts hemorrhagic transformation and poor outcomes after acute anterior stroke. Eur J Neurol. 2018;25(12):1432-e1122.

40. Rawshani A, Rawshani A, Franzen S, Sattar N, Eliasson B, Svensson AM, Zethelius B, et al. Risk factors, mortality, and cardiovascular outcomes in patients with type 2 diabetes. N Engl J Med. 2018;379(7):633-644.

41. Testai FD, Hillmann M, Amin-Hanjani S, Gorshkova I,
Berdyshev E, Gorelick PB, Dawson G. Changes in the cerebrospinal fluid ceramide profile after subarachnoid hemorrhage. Stroke. 2012;43(8):2066-2070.

42. Arboix A, Garcia-Eroles L, Massons J, Oliveres M. Predictive clinical factors of in-hospital mortality in 231 consecutive patients with cardioembolic cerebral infarction. Cerebrovasc Dis. 1998;8(1):8-13.

43. Hannun YA, Luberto C, Argraves KM. Enzymes of sphingolipid metabolism: from modular to integrative signaling. Biochemistry. 2001;40(16):4893-4903.

44. Mullen TD, Hannun YA, Obeid LM. Ceramide synthases at the centre of sphingolipid metabolism and biology. Biochem J. 2012;441(3):789-802.

45. Mohamud Yusuf A, Hagemann N, Hermann DM. The acid sphingomyelinase/ ceramide system as target for ischemic stroke therapies. Neurosignals. 2019;27(S1):3243. 